\title{
Logística: punto de inflexión del mercado moderno
}

\author{
Julieth Cecilia Álzate Alvarán ${ }^{1}$ \\ Shary Janice Pérez Olascuaga ${ }^{2}$
}

\section{Resumen}

En el presente capitulo se expone la revisión bibliográfica sobre el rol de la logística en el logro de la competitividad en las organizaciones modernas. Identificar la influencia que ha logrado la gestión logística es un aporte para mostrar la relevancia que debe dársele para dejarla de identificar con meras acciones de trasporte y/o suministros, resaltando el papel preponderante en la concesión de ventajas competitivas, valor agregado y mejora en tiempos y costo que redundan en la satisfacción del cliente final y de la misma empresa al ver los productos o servicios en óptima calidad en mano de para quienes fueron creados, así como rentabilidad y crecimiento interno.

\section{Palabras Claves}

Supply Chain, Logística, Competitividad, Cadena de Suministro.

\begin{abstract}
In this chapter, the bibliographic review on the role of logistics in achieving competitiveness in modern organizations is presented. Identifying the influence that logistics management has achieved is a contribution to show the relevance that must be given to stop identifying it with mere transport actions and / or supplies, highlighting the preponderant role in the granting of competitive advantages, added value and improvement in times and cost that result in the satisfaction of the final customer and the same company to see the products or services in optimum quality in hand of those for whom they were created, as well as profitability and internal growth.
\end{abstract}

\section{Keywords}

Supply Chain, Logistics, Competitiveness.

1. Magister en Administración, becaria del programa de formación de alto nivel del departamento de Sucre. Alcaldía de Sincelejo (Sucre).

2. Magister en Negocios Internacionales, becaria del programa de formación de alto nivel del departamento de Sucre. Especialista en docencia mediada por TIC'S. Gobernación de Sucre. 


\section{Introducción}

La evolución constante del mercado de bienes y servicios ha posibilitado que los diversos actores y variables que mueven dicho sistema estén en constante cambio. Uno de ellos y quizás cuya evolución ha sobresalido es la logística, la cual paso de ser vista como un proceso externo de las organizaciones y sus productos a convertirse en punto vital para el logro de las metas comerciales y de posicionamiento. Las operaciones logísticas, el proceso que ello implica en toda la cadena de suministros son hoy vitales para el éxito de una organización en cualquier sector desde donde se mire.

Señalar el rol de la logística en la empresa moderna es como hacer una radiografía a la estructura que atraviesa todos los procesos de la empresa, o por lo menos esta es la meta de la logística moderna, puesto que actualmente la gestión de la misma es un proceso integrador que repercute en el cliente y en la tan anhelada competitividad de las organizaciones. En consecuencia planear y ejecutar una logística integral, cíclica y permanentemente monitoreada aporta un sustrato invaluable para las organizaciones que quieren avanzar en el mercado global, donde la exigencia en tiempo y costos es cada vez mayor.

\section{Discusión}

Planear, implementar y controlar eficientemente el flujo y almacenamiento de materia prima, el inventario en proceso, productos terminados y su información relacionada desde el origen hasta el punto de consumo, en forma eficiente $y$ al menor costo posible, para satisfacer los requerimientos de los clientes es la conceptualización que el Council of Logistics ManagementCLM (1986),(Conocido actualmente como Council Logistics Management Professionals - CSCMP), hace sobre el concepto de logística. Sin embargo, Sarria (2012) señala que el significado de logística fue en principio un término asignado a área militar, cuyo propósito era asegurar el movimiento de los recursos necesarios para la guerra, pero que posteriormente empezó a aplicarse en el ámbito empresarial como el nuevo escenario para el desarrollo de técnicas y habilidades para facilitar el flujo de bienes y servicios desde quienes los producen hacia los consumidores finales.

Este cambio generó desarrollo y organización a las empresas que apostaron a la implementación logística; tanto así que esta ha evolucionado a todo nivel de la organización, ampliando su espectro de acción y dejando de ser el último eslabón del proceso productivo. En este sentido, el Ministerio de Industria, Turismo y Comercio de España (2007) expone que la logística dejó de ser el proceso para tener un producto en un momento específico en determinadas condiciones y lugar, para ser aplicada a todo el proceso de producción "desde su inicio hasta su llegada al consumidor y su posible devolución o reciclaje" (p. 8)

Servera (2010) plantea que desde el mundo empresarial la logística era asumida como un proceso sin capacidad de generar valor, mientras que en los centros de conocimiento como el área para la creación de modelos técnicos que facilitaran la distribución de acuerdo a las características propias de un producto. El autor señala que los postulados del marketing hicieron revalorar la importancia de la logística orientándola hacia el papel en la satisfacción del cliente, teniendo en cuenta la premisa de que la entrega del producto era igual de fundamental que su elaboración y calidad para el logro de la satisfacción a plenitud del cliente.

La apertura comercial mundial ha hecho que las empresas canalicen muchos esfuerzos en llegar más rápido al cliente final y a mayores cantidades y mayores distancias en el menor tiempo posible, cumpliendo las condiciones de calidad y buscando la satisfacción del destinatario. En este sentido la logística ha tomado un papel preponderante en el logro de la tan anhelada competitividad; así lo registran Ballesteros \& Ballesteros (2004) al indicar que la logística ayuda al logro de la competitividad mediante altos niveles de servicio a bajo costo.

La logística como proceso es ahora vista de un modo integral y por ello la implementación y análisis de la misma en una organización debe Anfibios ISSN: 2665-1513 
ser del mismo modo para lograr convertir este proceso en una ventaja competitiva. Este punto fue estudiado por el Ministerio de Industria, Turismo y Comercio de España (2007) en Pyme con el fin de identificar prácticas logísticas en la cadena de suministros (diseño, aprovisionamiento, producción, almacenamiento, transporte y distribución y servicio al cliente). Una vez analizado dichos aspectos el estudió concluyó que la gestión logística reviste gran importancia para lograr la competitividad a nivel nacional e internacional. Hacen énfasis en el uso racional y flexible de los recursos logísticos con el propósito de obtener mejores tiempos de entrega y calidad óptima en el servicio.

Desde la necesidad competitiva de una organización la planeación del proceso logístico y la permanente evaluación del mismo permite la implementación rápida de acciones de mejora y hasta la identificación de posibles fallas, antes de que ocurran. Para efectuar esto Ballesteros y Ballesteros (2004), luego de un análisis en potencias mundiales en materia logística ( apón, Francia, Estados Unidos y Alemania), plantean que el seguimiento de una serie de indicadores proporciona la información necesaria para la toma de decisiones en el ámbito de la cadena logística y de suministros, Dichos indicadores son el inventario promedio/Ventas, el costo logístico/ventas, las oportunidades en los aprovisionamientos, en los suministros a los clientes contra pedidos y a los clientes contra almacén, así como también los suministros perfectos de los proveedores y de pedidos perfectos a los clientes, la utilización de las capacidades de producción, las capacidades de la cobertura del inventario de productos terminados y la cobertura del inventario de materia prima y materiales.

Uno de los criterios que actualmente exigen los mercados internacionales a las empresas para que estas se diferencien entre el gran cumulo de organizaciones que compiten es la generación de valor en el producto o servicio que ofrecen. Crear valor implica por sí mismo un proceso de innovación, transferencia de conocimiento y aplicación de tecnologías; en este sentido y siendo la logística parte integral de la cadena de suministros de las empresas altamente compeEdiciones EFIM titivas debe esta también aportar a la creación, consolidación y conservación del valor del producto y/o servicio.

Esta visión integradora es posible haciendo que la logística esté en todo el proceso. Así lo sostiene Ocampo (2009) al señalar que la función logística dejó de ser la entrega de bienes y pasó a ser un entramado que deja ver sus frutos en la confirmación de la satisfacción de las expectativas del cliente. El autor sostiene que la competitividad de un producto es posible si se suman en él la calidad intrínseca, el valor agregado y el proceso de mejoramiento continuo. Es en esta última donde la gestión logística integral actúa en mayor medida puesto que con ella se tiene control del proceso productivo "desde el proveedor del proveedor, hasta el cliente del cliente" (p. 117). Esta visión global que otorga la logística permite que las estrategias competitivas que aplique la organización estén engranadas con todo el aparato productivo, gerencial y comercial.

Es de subrayar que la implementación logística en una organización, sin importar su tamaño, permite optimizar cada uno de los procesos interno y externo. El propósito de esto es cumplir la promesa de valor hecha a los clientes, lo cual implica garantizar la calidad de lo que se entrega ya que resultaría absurdo considerar un "justo a tiempo" poniendo en duda la seguridad, fiabilidad y eficiencia de la cadena de suministro en la organización. Cuando se realiza la gestión apropiada en la logística se garantiza situar el producto en la zona correcta, en el momento adecuado y con las condiciones oportunas. Así pues, esto finalmente tendrá efectos directos en la calidad del servicio y en los costos del producto, lo que simultáneamente genera ventajas competitivas que hacen que los clientes tengan preferencia por aquella organización o producto que los satisface en el mejor y más amplio sentido.

Aunque el logro de la competitividad no cuenta con una fórmula mágica que se adapte a todas las organizaciones, puesto que cada una posé un ADN único que hace necesaria la adaptación de criterios y elementos del proceso, los estudios y publicaciones sobre el tema han centrado la 
atención en comprender y compendiar una serie de elementos claves para lograr la competitividad con base en un sistema logístico. Tal es el caso de Cooltra Blog (2016) donde se identifican cuatro elementos fundamentales en la gestión logística para la competitividad. Estos elementos son el establecimiento de un plan de compras o producción, el manejo de los inventarios, los métodos de transporte y distribución y la atención al cliente.

Los anteriores elementos permiten concluir que el cliente es la base del sistema logístico y que para la satisfacción de este trabaja todo el andamiaje corporativo, por lo que finalmente cuando se realiza la gestión apropiada en la logística de los productos se está garantizando situar este en sitio deseado por el cliente y la empresa. En este sentido, Chávez (2012) le apuesta a plantear el proceso logístico como derrotero de la organización para logra la diferenciación entre las otras empresas del sector al que pertenezca. El autor asegura que la logística brinda oportunidades diversas para tener una posición relevante en el mercado en temas de tiempos, uso recursos y calidad en la entrega.

Sean pequeñas, medianas o grandes, las empresas han identificado en la logística un factor determinante para llegar al cliente en términos de calidad. Es por ello que las operaciones logísticas ya no son vistas como un deber si no como una estratega diferenciadora que crea valor al negocio, es decir un elemento de competitiva que puede catapultar a la organización si es gestionado, supervisado y evaluado constantemente. El logro de esto es un proceso sistemático en el que la organización debe apostar con recursos, tiempo y talento humano, tal como lo señala Entrepreneur (2017) al considerar que las empresas de este siglo deben tener en cuenta que la tecnología es un elemento que debe estar presente en su operación logística como forma fundamental y rápida de conocer los datos del proceso para generar actuaciones prontas en temas de entrega, inventarios, puntos de venta, transporte con apuestas al de crossdocking y/o multimodal, así como la trazabilidad desde el inicio del proceso hasta su culminación y la también denominada logística inversa .
Respecto a la entrada en escena de la logística inversa es preciso señalar la importancia que ha venido cobrando en los últimos años por su aporte a la interacción responsable de la empresa con el contexto y el medio ambiente. Sobre el particular, Silva (2015) propone un reflexión en la que señala que el mismo término de logística propuesto por la CSCMP integra el término de "inverso" como parte de la gestión logística, es decir que involucra las acciones de sostenibilidad, recuperación ecológica mediante acciones concretas como "reutilización, reventa, reparación, remanufactura, reciclaje y rediseño" ( $p$. 27).

Por otro lado, Ytoshi, Giro \& Dos Santos (2010) explican que la logística inversa reviste un proceso de planificación y control de elementos sustanciales del proceso productivo tales como las materias primas, así como también del inventario de productos, productos terminados y la información del proceso integralmente hasta llegar a la disposición final del producto y la forma como en ello se encuentra valor significativo para el reprocesamiento y hasta para el mismo cliente que requiere dichos servicios. Los autores sostienen que la logística inversa es una de las innovaciones que se han integrado a los modelos logísticos como forma de generar valor al producto, aumentar las capacidades competitivas y como factor diferenciador ante el cliente al tiempo que influencia de manera directa en los costos.

Por lo anterior la logística llega a convertirse en una ventaja competitiva para las empresas a través de la reducción de costos y dándole valor agregado para el cliente final, es decir, ofreciendo un plus diferenciador en sus productos, lo cual solo es posible poniendo en marcha herramientas para la optimización de resultados como el uso de la tecnología, gestión de información, optimización en la cadena de suministro. En tal medida, que una estrategia logística competitiva es materia de ideas, conocer, manejo de información y de relaciones y sobre todo la decisión de encarar retos de constante cambio producto del entorno en el cual se maneja en bien que ofrece la compañía. 
Un aporte adicional lo hace Monterroso (2000) al plantear algunas herramientas para mejorar la competitividad con las cuales estima se podría lograr una mayor participación de la empresa en el mercado, aumento en las utilidades y así mismo un mayor potencial de crecimiento. Entre dichas herramientas destaca las siguientes:

- Justo a tiempo: un concepto que considera la eliminación de desperdicios, cuya característica es la operatividad con bajos inventarios, pocos proveedores, flexibilidad en las operaciones enfocándose siempre en el cliente.

- Reingeniería: administración de proceso en vez de funciones, buscando óptimos niveles de eficiencia.

- Administración Total de la Calidad o Total Quality Management (TQM): tienda a ser un sistema de prácticas que persiguen "cero defectos", producción agilizada y optimización en los tiempos de respuesta.

- Respuesta Eficiente a consumidor o Efficiente Consumer Response (ECR): integración de proveedores y clientes a través los procesos logísticos y manejo de la información para obtener mayor eficiencia en toda la cadena de productos masivos.

- Tercerizacion de la distribución: a través de la tercerización con empresas especializadas de distribución y manejo de productos se pretende aumentar la eficiencia.

- Crossdcking: reducción de costos y un menor lead time total a través de la distribución y entrega de productos sin previo almacenamiento.

- Tecnología de grupos: manufacturación en flujo en línea recta, buscando acelerar procesos y reducción de inventarios en procesos.

- Postponement: dar rápida respuesta a las necesidades de los clientes, a través de un retraso en el agregad diferenciador de los productos, ubicándose esto en la fase final de la producción.

Las anteriores herramientas están enfocadas a la satisfacción del cliente, aumentar la eficacia, están orientadas a los procesos y la disminución de desperdicios, lo cual persigue la competitividad de la organización. Es por ello que la logística se convierte en un factor fundamental Ediciones EFIM para apostar a la competitividad en los mercados nacionales o internacionales. Teniendo claro, que el buen desempeño logístico conjuga variables como la infraestructura, competitividad y calidad en servicios de transporte; eficiencia y eficacia en aduanas y puertos; capacidad de seguimiento y localización de mercancías entre otros, lo cual permite perfeccionar los tiempos y así mismos los costos de transporte, almacenamiento y distribución, desde la fase de abastecimiento hasta el consumidor final como lo señala Ballesteros (2016).

Acorde a lo indicado se identifica que la logística es un proceso en el cual influyen diversas variables, las cuales es necesario monitorear mediante herramientas pertinentes y ligadas a la tecnología para la mejora de los tiempos de reacción y la propuesta de mejoras continuas de acuerdo con los cambios que se vayan suscitando en el mercado, en el producto o al interior de la empresa o a las anticipaciones que logre efectuar la misma sobre el panorama y contexto del negocio.

En este aparte abordaremos la importancia del mejoramiento continuo en la logística como un eje esencial para lograr, mantener o mejorar los niveles de competitividad. Es claro que los mercados y los clientes están en evolución permanente y rápida lo cual obliga a las empresas a analizar el modelo logístico que aplican y consultar permanentemente que nuevos esquemas se están apropiando en la competencia, pero más aún a construir modelos propios donde la innovación, la tecnología, la gestión del conocimiento y las características internas y del negocio sean los factores que determinen dicha apuesta e implementación, es decir que no sea la aplicación de modelos externos que no engranan a la perfección con las características propias.

En esta línea de mejoramiento logístico con base en las lo intrínseco de la organización Novoa \& Sepulveda (2008) abordaron la investigación de la industria de empaques y envases de Colombia, asociadas a Acoplasticos. Para ello efectuaron un análisis integral del funcionamiento de la operación logística con base en la aplicación de una encuesta empresarial y entrevistas en 
profundidad. Como resultados identificaron que el sector registraba ineficiencia en la gestión logística en aspectos fundamentales como la integralidad del proceso, puesto que es resorte de áreas específicas y no transversales como es el deber ser de la misma. Otro de los aspectos son la poca sistematización y organización de las cifras del sector lo cual dificulta los procesos de análisis histórico para la visualización de metas alcanzadas y mejoras a desarrollar.

Por consiguiente los autores proponen alternativas de mejoramiento encaminadas a aunar esfuerzos para la consolidación de un modelo logístico propio y solido basado en el trabajo en equipo, esto mediante el uso de las siguientes estrategias: "Comité permanente de logística, gestión gremial de Acoplásticos, trabajo interno de las empresas del sector y benchmarking y seguimiento de la actividad logística" (p.57). El Centro de Innovación y Desarrollo Empresarial -CIDEM (2004) sostiene que existen varios factores de cambio que se deben generar para la mejora sustancial del proceso y gestión logística con miras a avances competitivos representativos. Entre estos factores mencionan la importancia de la reestructuración interna y la apropiación de la logística como "redes innovadoras y actuar como facilitador de cambios" (p.94) lo cual repercute en una visión conjunta del proceso.

Otro factor es la construcción de asociaciones logísticas con otros miembros del sector y recalca el uso de sistemas de información como una herramienta fundamental para el monitoreo del proceso y el compendio y análisis de forma ágil de la información del mismo, lo cual repercute en toma de daciones más acertadas y basadas en datos reales y pertinentes.

Es por ello que las necesidades de mejorar o implementar el proceso logístico deben partir del análisis sistemático de las necesidades propias, es decir descubrir las deficiencias y plantear acciones concretas de mejoramiento. Al respecto, Giannice (2013) subraya que la logística es la fibra central de todo modelo de negocio debido a que mediante ella se logra la relación con los clientes y la fidelización de los mismos, así como la entrega a ellos de diferenciación.

\section{Conclusión}

La puesta en marcha de la gestión logística en las organizaciones a tono nivel constituye un factor diferenciador que marca un punto de inflexión en la linealidad productiva a la que el mundo estaba acostumbrado. Es por ello que la logística actual se reconoce como un proceso integral y cíclico que atañe a todas las áreas o sectores de una organización por lo que ha ido tomando una posición importante dentro de planeación y ejecución de la estrategia competitiva.

Ser competitivo en el mercado global es una meta que ha llegado recurrir a la capacidad de ingenio, imaginación y hasta a otros métodos menos ortodoxos, pero es la logística una de esas herramientas, que sin tanto misterio, contribuye al logro de metas, la obtención de información y la satisfacción del cliente, que es en última el deber ser de toda empresa. La competitividad es por consiguiente fruto de la creación y aplicación de buenas prácticas logísticas que son el resultado del conocimiento interno de la organización y el permanente monitoreo y análisis de la información que de ella se obtienen.

No existe una fórmula mágica generalizada para ser competitivo porque el material o insumos para lograrlo están al interior de cada empresa y apostar a dicha introspección y desarrollo de modelos logísticos propios aporta sin duda a la diferenciación e innovación que el mundo empresarial necesita para ser parte del mundo personal de cada cliente.

\section{Referencias bibliográficas}

Ballesteros, D., \& Ballesteros, P. (2004). La logística competitiva y la administración de la cadena de suministros. Scientia Et Technica, vol. X, núm. 24, mayo, 2004, pp. 201-206. Universidad Tecnológica de Pereira. Colombia

Ballesteros, J. (2016). Como la logística contribuye al desarrollo de la competitividad de una empresa. Retrieved from https://repository.libertadores.edu.co/bitstream/handle/11371/948/ PlazasGilJuanCarlos.pdf? sequence $=2$ \&is Allowed=y 
Centro de Innovación y Desarrollo Empresarial (CIDEM) (2004). Guías de gestión de la innovación producción y logística. ISBN 84-3936186-6. $1^{\text {a }}$ edición en Castellano.

Chávez, J (2012). Cadena de valor, estrategias genéricas y competitividad: El caso de los productores de café orgánico del municipio de Tanetze de Zaragoza, Oaxaca.

Competitividad, C. P. de. (n.d.). Informe Nacional de Competitividad 2017-2018. Retrieved from https://compite.com.co/wp-content/ uploads/2017/10/cpc_brochure.pdf

Cooltra Blog. (2016). La gestión logística. Retrieved from https://blog.cooltra.com/la-gestion-logistica-la-clave-de-la-competitividad/

Council of Logistics Management- CLM (1986).Libro de OAK "What's it all about".

Entrepreneur. (2017). 8 trucos de logística para ser competitivo. Retrieved from https://www. entrepreneur.com/article/267891

Giannice, S (2013). La logística y su esencia en los modelos de negocio. $1^{\circ}$ Congreso Internacional Sobre Nuevas Tendencias de la Logística Empresarial, Bogotá, Colombia. Noviembre 14-15.

Ministerio de Industria, Turismo y Comercio de España (2007). Logistica y competitividad de las Pyme

Monterroso, E. (2000). El proceso logístico y la gestión de la cadena de abastecimiento. Retrieved from https://www.researchgate.net/publication/296483187_El_proceso_logistico_y_la gestion_de_la_cadena_de_abastecimiento

Novoa, F., Sepulveda, P., (2008). Mejoramiento de la gestión logística de las empresas afiliadas a Acoplásticos: diagnóstico y recomendaciones. REVISTA Universidad EAFIT. Vol. 45. No. 153. 2009. pp. 38-61.

Ocampo, P. (2009). Gerencia logística y global Revista Escuela de Administración de NegoEdiciones EFIM cios, núm. 66, pp. 113-136. Universidad EAN. Bogóta, Colombia

Sarria, M. (2012). LA_LOGISTICA_COMO HERRAMIENTA_DE_COMPETI. Retrieved from http://www.academia.edu/21819140/LA LOGÍSTICA_COMO_HERRAMIENTA_DE_ COMPETITIVIDAD_PARA_LAS_EMPRESAS

Servera, D. (2010). Concepto y evolución de la función logística. INNOVAR. Revista de Ciencias Administrativas y Sociales, vol. 20, núm. 38, pp. 217-234. Universidad Nacional de Colombia. Bogotá, Colombia

Silva, A. (2015). Importance of reverse logistics and their impact on the environment. TAU e-Journal of Multidisciplinary Research. Trabajo de investigación desarrollado en el marco del General Management Program with emphasis in Supply Chain Management \& Logistics (2015). CFMD deTecana American University (TAU), USA.Vol.: 5, Nro.:1

Ytoshi, F., Giro, R., \& Dos Santos, M (2010). Logistica reversa e a sustentabilidade empresarial. XIII Seminario en Administración. ISSN 2177-3866 